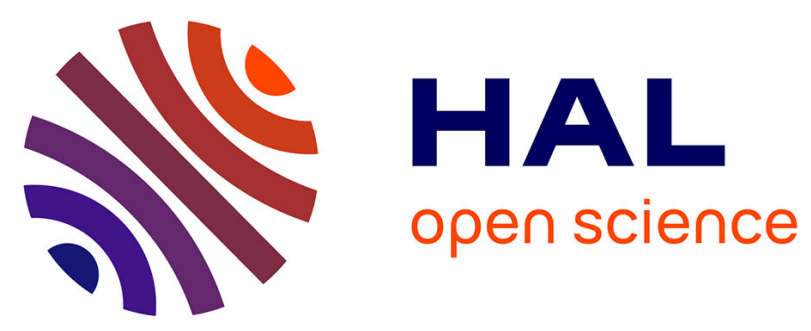

\title{
A 2.4 GHz 1-Dimensional Array Antenna driven by Vector Modulators
}

Nidaa Tohmé, Jean-Marie Paillot, David Cordeau, Sébastien Cauet, Yann Mahé, Patrick Ribardière

\section{- To cite this version:}

Nidaa Tohmé, Jean-Marie Paillot, David Cordeau, Sébastien Cauet, Yann Mahé, et al.. A $2.4 \mathrm{GHz}$ 1-Dimensional Array Antenna driven by Vector Modulators. IEEE MTT-S International Microwave Symposium, Jun 2008, Atlanta, United States. pp.803-806. hal-00683429

\section{HAL Id: hal-00683429 \\ https://hal.science/hal-00683429}

Submitted on 28 Mar 2012

HAL is a multi-disciplinary open access archive for the deposit and dissemination of scientific research documents, whether they are published or not. The documents may come from teaching and research institutions in France or abroad, or from public or private research centers.
L'archive ouverte pluridisciplinaire HAL, est destinée au dépôt et à la diffusion de documents scientifiques de niveau recherche, publiés ou non, émanant des établissements d'enseignement et de recherche français ou étrangers, des laboratoires publics ou privés. 


\title{
A 2.4 GHz 1-Dimensional Array Antenna driven by Vector Modulators
}

\author{
Nidaa Tohmé ${ }^{1}$, Jean-Marie Paillot ${ }^{1}$, David Cordeau ${ }^{1}$, Sébastien Cauet $^{1}$, Yann Mahé2 Patrick Ribardière $^{1}$ \\ ${ }^{(1)}$ University of Poitiers, LAII, 42 Av. du Recteur Pineau, 86022 Poitiers Cedex, France \\ Email:jmpaillot@,iutang.univ-poitiers.fr \\ ${ }^{(2)}$ University of Nantes, IREENA-Rue Christian Pauc, La Chantrerie, BP50609, 44306 Nantes Cedex 3, France
}

\begin{abstract}
This paper aims at developing an intelligent radio-communication prototype using multiple-antenna phased array whose bearing can be controlled electronically. Such a system has proved its quality in terms of gain and SNR improvement. The architecture presented in this article runs at $2.4 \mathrm{GHz}$ with a bandwidth channel of $11 \mathrm{MHz}$ which is well suited for WIFI 802.11b applications. This original system uses quadrature modulators as active phase-shifters, and noise sensitivity is studied and simulated to prove the interest of this architecture. A circuit is under tests and first elementary active parts RF measurements are given.
\end{abstract}

.Index Terms - Active arrays, Antenna array, Modulators, Phased array, Quadrature Phase Shift Keying.

\section{INTRODUCTION}

In transmission systems, omnidirectional antennas are widespread but cause an important Electro-Magnetic pollution. Indeed, omnidirectional antennas transmitters radiate uniformly and only a small fraction of this radiated power reaches the intended receiver. Consequently, in an environmental perspective, the efficiency between the useful power and lost power is inappropriate in many applications. The directivity of an antenna is a statement of how the RF energy is focused in one or more directions. Because the amount of RF energy remains the same, but is distributed over less area, the apparent signal strength is higher and increases the antenna gain. Electronically-steered phased arrays are an important technology used in defense applications and civil radio communications. Moreover, radio communications systems performances are improved by using active electronically scanned arrays. In such system, the directional sensitivity of the phased array can be adjusted by controlling the relative amplitudes and phases of signals applied on each pattern of the array.

Technical solutions, with phase-shifters or phase-shifterless, are proposed to perform this electronic beam steering. Even if the most common method is to use phase sifters, their losses are a main issue since it deteriorates the overall system performances. Others passive solutions such as Rotman lenses or Butler matrices are used, but only for arrays made of many patterns. On the other hand, active solutions, presented by R. York and al [1] [2], use coupled oscillators array to control the relative phases through adjustment of the oscillator natural frequencies. Since these seminal works, few papers present new investigations and improvements of this technique, in particular concerning the reduction of side lobes [3]. In this context, this paper presents an original architecture, using vector modulator, to perform electronic beam steering by controlling the relative phase and amplitude of signals applied on each pattern of the array.

After a brief review of beam steering of a one dimensional array presented in section II, the original phased array architecture is discussed in section III. In particular, the EVM and $B E R$ sensitivities to the random variations on the signal commands is studied. Finally, section IV shows the firsts measurements of the system at $2.4 \mathrm{GHz}$.

\section{GENERAL PRINCIPLE}

An intelligent antenna consists in an array of individual radiation elements (elementary patch antennas), which are placed in a particular configuration (linear, circular or matrix). By associating these elementary antennas in such arrays and by changing the characteristics of the associated signals, the array can present different gains according to the direction.

In this paper, only a uniform-spaced linear array of $\mathrm{N}$ elementary patch antennas placed at the same distance $d$ between them, is considered as shown in Fig.1.

Where $d$ is the distance between each patch, $\theta$ is the radiation angle. In order to study the behavior of this configuration, let us suppose that each elementary antenna is

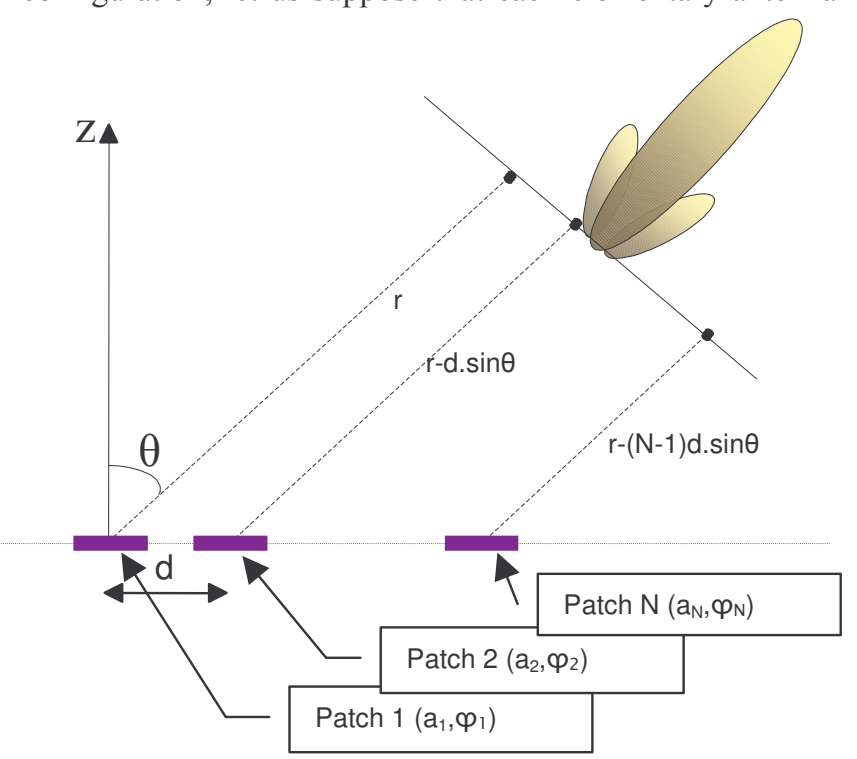

Fig. 1. $\mathrm{N}$ radiation antennas 
excited by harmonic signals at the same frequency, and multiplied by different complex coefficients:

$$
\mathrm{w}_{\mathrm{i}}=\mathrm{a}_{\mathrm{i}} \cdot \mathrm{e}^{\mathrm{j} \varphi_{\mathrm{i}}}, \quad \mathrm{i} \in[1 \ldots \mathrm{N}]
$$

In a first approximation, let us consider that the relative phase applied between two adjacent elements is the same $\varphi=\varphi_{i+1}-\varphi_{i}$. For far-field, the total electrical radiated field is the sum of individual field radiates by each elementary antenna. Thus, the total field is given by the following expression:

$$
\overrightarrow{\mathrm{E}}=\sum_{\mathrm{n}=1}^{\mathrm{N}} \overrightarrow{\mathrm{E}_{\mathrm{n}}}=\frac{A \cdot \mathrm{e}^{-\mathrm{j} \cdot \mathrm{k} \cdot \mathrm{r}}}{\mathrm{r}}\left[\sum_{\mathrm{n}=1}^{\mathrm{N}} \mathrm{e}^{\mathrm{j} \cdot(\mathrm{n}-1)(\mathrm{k} \cdot \mathrm{d} \cdot \sin \theta-\varphi)}\right]
$$

where $r$ is the maximum distance between the source and the observation plan and $k$ is the propagation constant.

According to (2), the radiation angle $\theta$ for the maximum field amplitude is given by :

$$
\theta=\operatorname{Arcsin}\left(\frac{\lambda \cdot \varphi}{2 . \pi \cdot d}\right)
$$

Thus, this direction depends on the relative phase applied between two adjacent elements. Furthermore, it could be shown that the optimal distance $d$ is $\lambda / 2$. In these conditions, Fig. 2 shows the normalized amplitude of radiated field versus the radiation angle $\theta$ for different values of $\varphi$.

As expected, the relative phase command allows to control the radiation angle and the directivity can be adjusted by varying the number $N$ of element. Indeed, with a eightelement array, the directivity is equal to $9 \mathrm{~dB}$ and the Half Power Beamwidth (HPBW) given by the following expression $2 * \arccos (1-\lambda / \mathrm{N} . d)$ is equal to $10^{\circ}$. On the other hand, a control of the amplitude $a_{i}$ applied on each element can ensure the null formation. For these reasons, to obtain an agile and electronically beam steering, it is essential to master these amplitudes and phases applied on each elementary antenna.
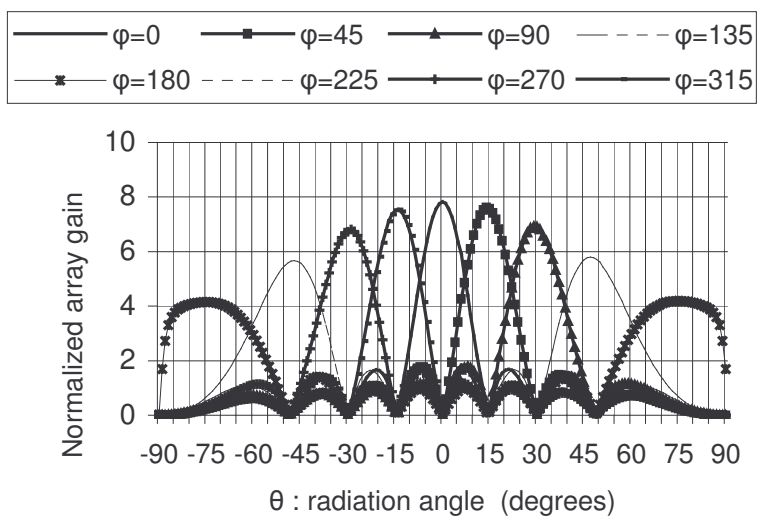

Fig. 2. Array gain for eight-element, with a measured elementary field of a patch.

\section{PHASED-ARRAY ARCHITECTURES}

If we consider only the phase-shifterless architectures, the main solutions are given below.

Recently, Ali Hajimiri and his work group proposed a solution using a polyphase VCO [4], able to generate LO frequency with $\mathrm{N}$ phases. In this architecture, the $\mathrm{N}$ phases are conveyed to each patch antenna via a distribution network and phase selectors ensure the required phase to each element. In this case, the phase variation is discrete, which does not constitute a problem while the discrimination steps are compatible with the application. Nevertheless, the distribution network of $\mathrm{N}$ phases constitutes here a real issue since each line must be forwarded to the phase selector in a symmetrical way.

Another solution, initiated by R. York [2], consists in coupling local oscillators between them in order to generate the different relative phases. It is a very interesting solution because it provides a continuous phase variation. However, this system seems to be relatively complex to implement and the theoretical maximum relative phase is equal $\pm 90^{\circ}$.

The original architecture presented in this paper uses vector modulators to achieve the relative amplitude and phase applied on antennas. Very few articles [5], [6] have been published on this solution which, however seems to be very interesting. Two configurations are possible, in the first one, the vector modulator can be placed on the LO path. In this case, the nonlinear and noisy behavior of this active phaseshifter does not affect directly the system performances. On the other hand, this configuration implies an up-conversion before antenna to transmit or receive the RF signal. Another solution is to place the vector modulator directly in the RF path. In these conditions, no up-conversion is required but the IQ modulators noise factors and the non linear behavior can affect the performances of the entire system. Furthermore, it seems to be interesting to study the degradation of the transmission quality according to the noise which affects the command signals.

To do that, system analysis allows to measure both bandwidth effects and impact of command noise on the constellations and BER. Advanced Design System software of the Agilent Society is used to perform this analysis. Fig.3 shows a simplified architecture, with four-elements, where the QPSK modulation is chosen as a source for its high sensitivity to phase noise. Symbol rate is set to $11 \mathrm{Mbit} / \mathrm{s}$, with a carrier frequency of $2.4 \mathrm{GHz}$. In this instance, the relative phase applied between each elementary antenna $\varphi$ is chosen equal to $80^{\circ}$. Consequently, the constellation rotation is also equal to $80^{\circ}$ as shown in Fig.4. This implies a radiation angle $\theta$ of $26.4^{\circ}$ according to (3). 


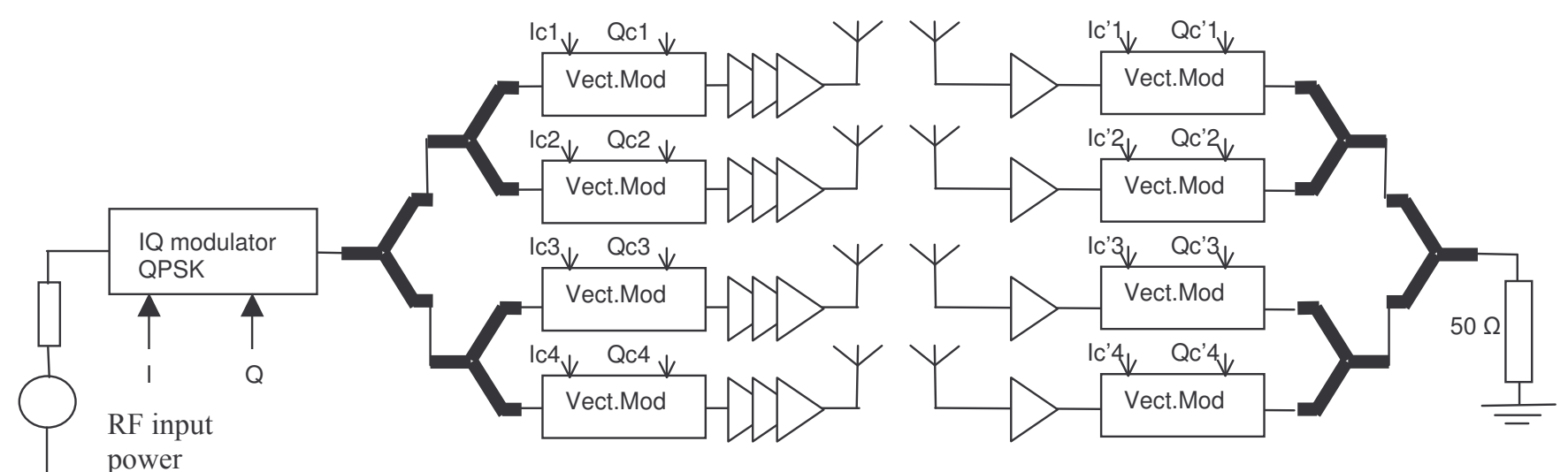

Fig. 3. Simplified phased array architecture

In this case, the noise factor of vector modulators is set to 2 $\mathrm{dB}$ but the command $\left(\mathrm{Ic}_{\mathrm{i}}, \mathrm{Qc}_{\mathrm{i}}, \mathrm{Ic}{ }_{\mathrm{i}}\right.$ and $\left.\mathrm{Qc}_{\mathrm{i}}{ }_{\mathrm{i}}\right)$ are noiseless. In the receiver, a dual architecture allows to recover the original constellation. The results are presented in Fig.5. Now, the objective is to analysis the noise effect on the system performances. To study the behavior versus the noisy command signals, noise sources have been placed on the Ic' ${ }_{i}$ and $\mathrm{Qc}_{\mathrm{I}}$ ' commands. Table1 shows EVM results for different noise values $\left(\mathrm{C}_{\min }\right.$ corresponds to the minimum value of the command voltages and $\mathrm{N}$ is the noise voltage). These EVM have to be added to those resulting from an error in the receiving angle given by Hajimiri [4]. In the same conditions, Fig.6 shows the BER evolution. With the above used conditions, it is possible to consider that a $(\mathrm{Cmin} / \mathrm{N})_{\mathrm{dB}}$ higher than $40 \mathrm{~dB}$ does not affect the performances in terms of $E V M$ and BER. However, these system simulations prove the

Const. $1 \bigcirc$ Const. $2 \square \quad$ Const. $3 \times \quad$ Const. $4 \nabla$

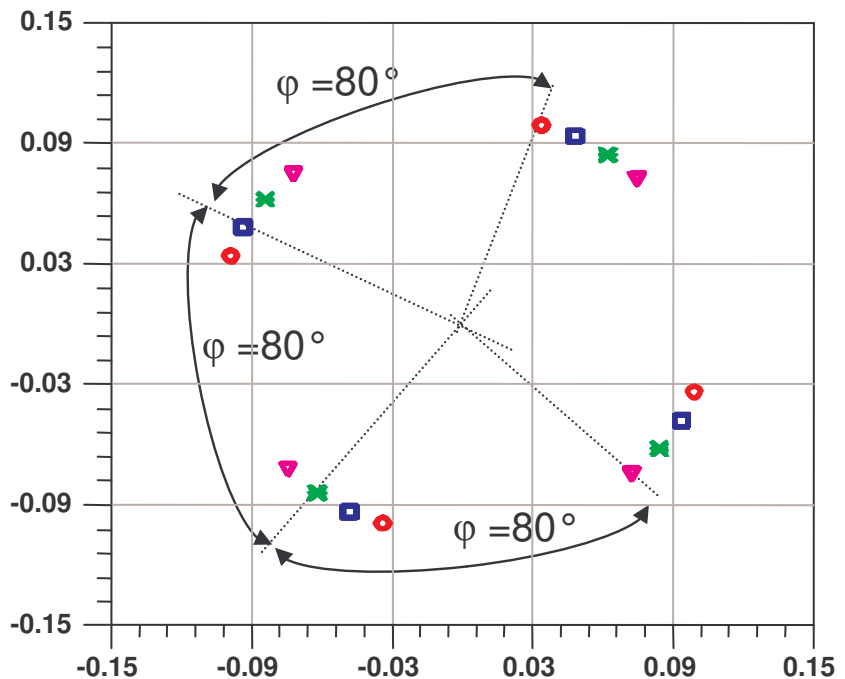

Fig. 4. Transmitted signal Constellation
TABLE I

SUMMARY OF EVM VERSUS COMMAND NOISE

\begin{tabular}{|c|c|}
\hline$\left(\mathrm{C}_{\min } / \mathrm{N}\right) \mathrm{dB}$ & $\mathrm{EVM}_{\max } \%$ \\
\hline 47.7 & 0.59 \\
\hline 39.7 & 0.72 \\
\hline 27.6 & 2.02 \\
\hline 19.6 & 5 \\
\hline 13.7 & 9.8 \\
\hline
\end{tabular}

necessity to take into account the noise which can be present on the command of the quadrature modulators used as active phase-shifter. Indeed, this command noise directly involves transmission perturbations. From this conclusion, a first design becomes possible.

Const. $1 \bigcirc$ Const. $2 \square \quad$ Const. $3 \times \quad$ Const. $4 \nabla$

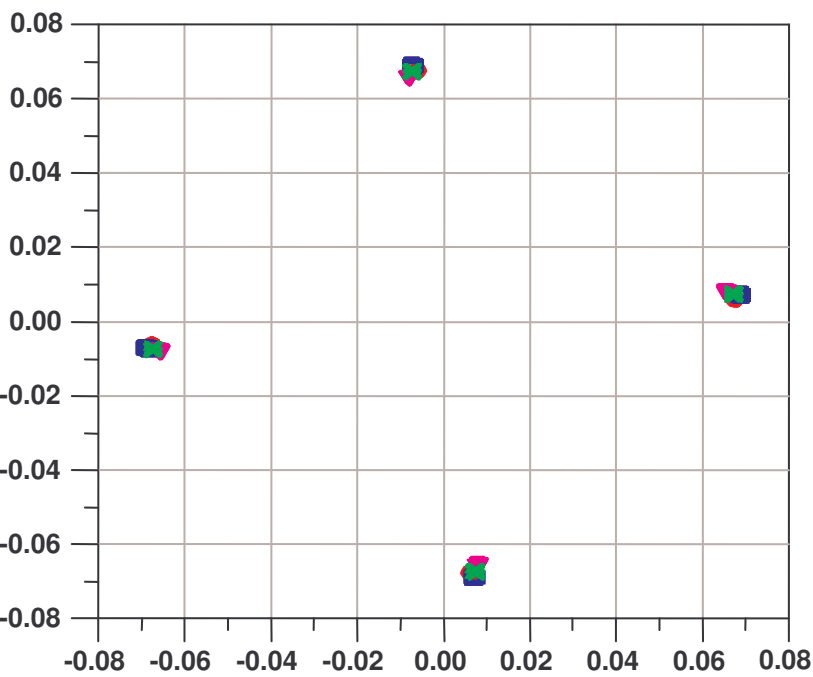

Fig. 5. Received signal Constellation 


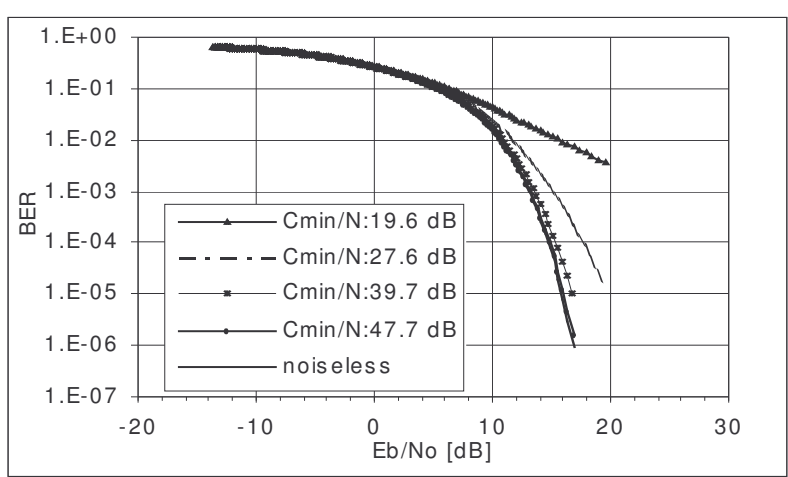

Fig. 6. BER versus Eb/No

\section{PRototype DESIGN}

This first design, with discrete components, is developing in our laboratory, with four elements patch antennas. The elementary command circuit (Fig. 7) uses an AD 8349 IQ quadrature modulator followed by an ADL5330 Voltage Gain Amplifier (VGA). Input and output 1:1 Transmission Line Transformers allow the single to differential mode transitions.

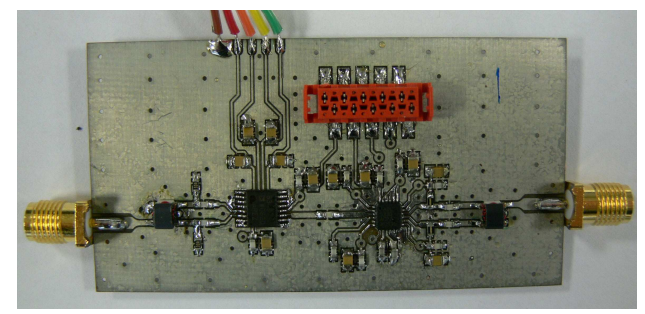

Fig. 7 Photograph of the active part

The output $1 \mathrm{~dB}$ compression point is equal to $0.2 \mathrm{dBm}$ for a VGA voltage control set at $1.4 \mathrm{~V}$. Note that, for this first approach the power amplifiers are not taken into account. "S" parameters are measured and $S_{11}$ and $S_{22}$ parameters are better than $-15 \mathrm{~dB}$.

Under these conditions, the $\mathrm{S}_{21}$ parameters have been

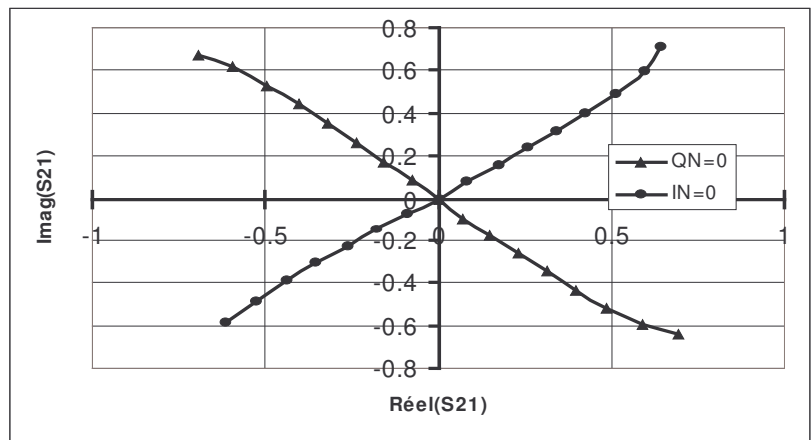

Fig. 8. Quadrature characteristics at $2.4 \mathrm{GHz}$ and $\mathrm{Vg}=1.2 \mathrm{~V}$. measured for the four elementary circuits, their behaviors are similar and one characteristic is given in Fig. 8. From these measurements, it is possible to calibrate the system for applying the commands $\mathrm{Ic}_{\mathrm{i}}$ and $\mathrm{Qc}_{\mathrm{i}}$ which will allow to obtain the required radiation angle $\theta$. Numerical system using FPGA has been performed to drive the different vector modulators.

Distribution path and elementary patch antenna have been developed at IREENA laboratory and work at a frequency of $2.4 \mathrm{GHz}$. The full system will be operational at the beginning of January and the first measurements of the sensitivity will begin as soon as possible.

\section{Conclusion}

An approach on phased array antenna command is analyzed. Electronically beam steering using vector modulator presents much advantages, in particular, an improvement of the SNR during the transmission, and a better repartition of the output power in the transmitter. This last one, can avoid the using of a high cost technology. System analysis confirm the interest of the architecture in taking in account the perturbations on the commands.

\section{ACKNOWLEDGEMENT}

The authors would like to thank M. Olivier Négro and P. Abbé for their technical contributions to this project and the helpful discussions.

\section{REFERENCES}

[1] R. A. York, R.C. Compton "Quasi-Optical Power Combining Using Mutually Synchronized Oscillator Arrays," IEEE Trans. Microwave Theory \& Tech., vol. 39, no.6, pp. 1000-1009 June 1991.

[2] J.J Lynch \& al., "Synchronization of Oscillators Coupled Through Narrow-Band Networks," IEEE Trans. Microwave Theory \& Tech., vol. 49, no.2, pp. 237-249 February 2001.

[3] T. Heath, "Simultaneous Beam Steering and Null Formation With Coupled, Nonlinear Oscillator Arrays," IEEE Trans. Antennas and Propagation, vol 53 no6, pp. 2031-2035, June2005.

[4] A. Natarajan, A. Komijani, A. Hajimiri, "A Fully Integrated 24-GHz Phased-Array Transmitter in CMOS," IEEE Journal of solid-state circuits, vol 40, no 12, pp. 2502-2514, December 2005.

[5] F. Ellinger, W Bächtod "Novel Principles for vector Modulator-based phase Shifters operating with only one control voltage" IEEE Journal of solid-state circuits, vol. 37, no 10, pp1256-1259, October 2002.

[6] S. Alalusi, R. Brodersen, "A $60 \mathrm{GHz}$ Phased-array in CMOS," IEEE Custom Integrated Circuit Conference (CCIC) 2006. 